$\begin{array}{lllllllllllllll}\text { A T A C H M I C A S C A N D I N A V I C A } & 19 & (1965) & 53-64\end{array}$

\title{
A Polarimetric Investigation of the Complex Formation between Zinc and Mandelate Ions
}

\author{
RAGNAR LARSSON and BÖRJE FOLKESON \\ Department of Inorganic Chemistry, Chemical Institute, University of Lund, Lund, Sweden
}

\begin{abstract}
The optical rotation of the L-mandelate ion has been measured for zinc-mandelate solutions of various composition. The measurements were made at 589, 578, 546, 436, and $365 \mathrm{~m} \mu$. From these data the following stability constants were obtained: $\beta_{1}=30 \mathrm{M}^{-1}, \beta_{2}=$ $260 \mathrm{M}^{-2}$, and $\beta_{3}=3870 \mathrm{M}^{-3}$. The molar rotations were calculated for the free ligand and for the three complexes (Table 7). These values fitted the Drude equation and the following values were obtained for the wavelength of the electronic transition responsible for the optical activity of the free ligand and the complexes: $\lambda_{0 \mathrm{~A}}=$ $230 \mathrm{~m} \mu: \lambda_{01}=210 \mathrm{~m} \mu \lambda_{02}=255 \mathrm{~m} \mu ; \lambda_{03}=215 \mathrm{~m} \mu$.
\end{abstract}

$A s$ is well known, coordination chemistry owes much to polarimetry. It $\mathrm{A}_{\text {was }}$ through the successful resolution into optical isomers of hexacoordinated, inert complexes that Werner's theory was firmly established. However, the emphasis of polarimetric studies has remained on the inert complexes through the years. Only now and then have investigations on labile complex systems been reported, mostly of a qualitative kind.

During the past two decades the application of general methods for the elucidation of the composition of complexes in solution has yielded paramount results. In the field of spectrophotometry, e.g., the exchange of the Job method for more exact treatments has been of great value. ${ }^{1}$ The use of measurements of optical rotation (OR), however, does not seem to have attracted the attention of workers in this field.

The availability of commercial, electrically recording polarimeters has encouraged us to apply OR-measurements to the study of the stepwise formation of complexes in solution.

We will closely follow the theory indicated by Fronæus ${ }^{2}$ who, to the best of our knowledge, was the first to give a general description of the use of OR-measurements for the above-mentioned purpose.

The system studied by Fronæus, ${ }^{2}$ copper(II)-tartrate, was found to be a very complicated one because of the formation of polynuclear complexes. This obstructed the complete interpretation of the data greatly. In order

Acta Chem. Scand. 19 (1965) No. 1 
to avoid this complication we have chosen as the ligand an ion of such structure that there will be very little chance of metal-ligand-metal bridges. From measurements on glycolate systems it is evident that this ion acts as a monodentate or a chelating ligand..$^{2-4}$ For systems where polynuclear complexes caused by hydrolysis of the metal ions could be suppressed, no indication of polynuclear products has been found. Especially the fact ${ }^{2,3}$ that both potentiometric and spectrophotometric investigations gave concordant results favours such an interpretation.

It then seems reasonable that the mandelate ion, which resembles the glycolate one but for the exchange of one hydrogen atom for a phenyl group introducing asymmetry, should also have a very slight tendency to act as a bridging ligand. Very few investigations of the complex chemistry of this ion can be found in literature, however, and none so detailed that the question of its bridging tendency is touched upon.

Hence, from the analogous reasoning above we have taken the $L_{-}$-mandelate ion as a ligand that should act well to test the method of OR-measurements in the investigation of equilibria of labile complexes. In order to avoid, in the first instance at least, complications due to Cotton effects in the used spectral range we have chosen zinc as the metal the complexity of which should be investigated.

\section{PRINCIPLE OF INVESTIGATION AND GENERAL FORMULAE}

The method of investigation implies that there is a change in the molar rotation of the ligand when it is bound to a metal ion and when in free form. Therefore, the difference between the optical rotation of mandelate solutions with and without metal perchlorate has been determined. In order to minimize variations of activity coefficients all solutions have been given an ionic strength $=2 \mathrm{M}$, by the addition of sodium perchlorate. In order to depress the hydrolysis of the metal, $\mathrm{pH}$ of the solutions was kept $\approx 5$ by using mandelatemandelic acid buffers with the concentration ratio 100: 1 . The concentration of mandelic acid was kept this low, so that the rotation of this species should be a small correction only.

At $\mathrm{pH}=5$ the amounts of hydrolytic products ${ }^{5}$ should be $<10^{-3} \%$ for all metal concentrations used in this work.

The solutions investigated thus have the general composition:

$$
C_{\mathrm{A}}{ }^{\prime} \mathrm{M} \mathrm{NaA}, C_{\mathrm{HA}} \mathrm{M} \mathrm{HA}, C_{\mathrm{M}} \mathrm{M} \mathrm{M}\left(\mathrm{ClO}_{4}\right)_{2},\left(2-C_{\mathrm{A}}{ }^{\prime}-3 C_{\mathrm{M}}\right) \mathrm{M} \mathrm{NaClO}_{4} \text {. }
$$

Denotations not stated explicitly are those usually employed in this laboratory (cf. Refs. 2, 6).

If $\vartheta^{\prime}$ is the angle that such a solution rotates the plane of polarized light, one obtains the following relation:

$$
\vartheta^{\prime}=\delta_{\mathrm{HA}}[\mathrm{HA}]+\delta_{\mathrm{A}}[\mathrm{A}]+\sum_{n=1}^{N} \delta_{n}\left[\mathrm{MA}_{n}\right]
$$

Here $\delta_{\mathrm{HA}}, \delta_{\mathrm{A}}$, and $\delta_{n}$ are the molar rotations of the acid, free ligand, and the $n$th complex, respectively. 
[HA] and $C_{\mathrm{A}}$ were corrected for the protolysis of the acid $[\mathrm{HA}]=C_{\mathrm{HA}}-\left[\mathrm{H}^{+}\right]$ $C_{\mathrm{A}}=C_{\mathrm{A}}^{\prime}+\left[\mathrm{H}^{+}\right]$

As this correction was of any importance only for very small values of $C_{\mathrm{A}}$ and $C_{\mathrm{HA}}$ an average of literature data ${ }^{7,8}$ of $\mathrm{p} K_{\mathrm{a}}=3$ was used. By subtracting $\delta_{\mathrm{HA}}[\mathrm{HA}]$ from (1) one obtains

$$
\vartheta=\delta_{\mathrm{A}}[\mathrm{A}]+\sum_{n=1}^{N} \delta_{n}\left[\mathrm{MA}_{n}\right]
$$

This operation is correct only if the mandelic acid does not form complexes with zinc. That this is not the case is indicated by measurements described below.

By close adherence to the method developed by Fronæus, ${ }^{2}$ we form the expression|

$$
\varphi=\left(\vartheta-\delta_{\mathrm{A}} C_{\mathrm{A}}\right) C_{\mathrm{M}}^{-1}
$$

Introducing the stability constants $\beta_{n}$ of the mononuclear complexes into (3), we obtain under the assumption that no polynuclear complexes exist:

$$
\varphi=\mathrm{X}^{-1} \sum_{n=1}^{N} \delta_{n} \beta_{n}[\mathrm{~A}]^{n}-\delta_{\mathrm{A}} \bar{n}
$$

Here $\mathrm{X}=1+\sum_{n=1}^{N} \beta_{n}[\mathrm{~A}]^{n}$

$$
\text { and } \bar{n}=\left(C_{\mathrm{A}}-[\mathrm{A}]\right) C_{\mathrm{M}}^{-1}=\mathrm{X}^{-1} \sum_{n=1}^{N} n \beta_{n}[\mathrm{~A}]^{n}
$$

It is evident that $\varphi$, under the assumptions made, is a function only of the free ligand concentration [A]. This means that $\bar{n}$ may be determined by measuring $\varphi$ for some values of $C_{\mathrm{M}}$. For a series of constant values of $\varphi$ and thus constant values of $[\mathrm{A}]$ one then obtains

$$
\bar{n}=\left(\delta C_{\mathrm{A}} / \delta C_{\mathrm{M}}\right)_{[\mathrm{A}]}
$$

In practice $C_{\mathrm{A}}$ was plotted against $C_{\mathrm{M}}$ and $\bar{n}$ was obtained as the slope of the straight lines that were obtained. The corresponding value of [A] was obtained as the intercept on the $C_{\mathrm{A}}$-axis. In this way corresponding values of [A] and $\bar{n}$ are obtained and from these the stability constants can be determined.

\section{EXPERIMENTAL}

All measurements were made with a Perkin Elmer Model 141 photoelectric polarimeter. This instrument can be read to $0.001^{\circ}$. The cells were of $10 \mathrm{~cm}$ lengths and termostated to $20.0^{\circ} \mathrm{C}$. Two cells were used, the exact lengths of which were related to one another by measuring the rotation of a sample with large rotation. The reported values are the mean of the readings of the two cells.

The polarimeter was equipped with a sodium lamp and a mercury one. With the help of a coloured glass filter and interference filters, respectively, the following lines could be isolated: $589,578,546,436$, and $365 \mathrm{~m} \mu$. The measurements were performed at all these five wavelengths.

Chemicals. Two different preparations of L-mandelic acid were used, from May \& Baker and L. Light \& Co, Ltd, respectively. The purity of the preparations was checked polarimetrically. The specific rotations were found to agree well with literature data.

$$
\begin{array}{ll}
{[\alpha]_{589}=-153.9 \text { and }-153.8, \text { resp. }} & \left(c f .-152.9^{\circ}\left(25^{\circ} \mathrm{C}\right)^{9}\right) \\
{[\alpha]_{546}=-185.3 \text { and }-185.1, \text { resp. }} & \left(c f .-183.5^{\circ}\left(25^{\circ} \mathrm{C}\right)^{9}\right)
\end{array}
$$

Acta Chem. Scand. 19 (1965) No. 1 
From these preparations sodium mandelate was made by neutralising a known amount of acid with carbonate-free sodium hydroxide. The solution was slowly evaporated to dryness, the salt was recrystallized, washed with alcohol and ether and the specific rotation was measured.

$$
\begin{array}{ll}
{[\alpha]_{580}=-102.8^{\circ}} & \left(-100.6^{\circ}\left(25^{\circ} \mathrm{C}\right)^{\circ}\right) \\
{[\alpha]_{640}=-123.2^{\circ}} & \left(-120.3^{\circ}\left(25^{\circ} \mathrm{C}\right)^{\circ}\right)
\end{array}
$$

Sodium perchlorate was prepared from analytical grade sodium carbonate and perchloric acid and recrystallized according to the prescription of Fronæus (Ref. 2, p. 31).

Zinc perchlorate was prepared from analytical grade zinc oxide and perchloric acid. From the recrystallized salt a stock solution was made, the concentration of which was determined by EDTA-titration. The excess of free acid was found to be $0.3 \%$ of the zinc concentration by means of a cationic exchange-alkalimetric titration. As this is close to analytical error, any excese of free acid has been neglected in the calculations.

\section{MEASUREMENTS}

In order to check that the free acid does not coordinate to the metal ion the rotation was measured for solutions of the composition: $C_{\mathrm{HA}} \mathrm{M} \mathrm{HA}, C_{\mathrm{M}}$ $\mathrm{M} \mathrm{Zn}\left(\mathrm{ClO}_{4}\right)_{2},\left(2-3 C_{\mathrm{M}}\right) \mathrm{M} \mathrm{HClO}_{4}$. In one series of solution $C_{\mathrm{M}}$ was kept constant and $C_{\mathrm{HA}}$ was varied, in another series $C_{\mathrm{HA}}$ was constant and $C_{\mathrm{M}}$ was varied. In both cases (cf. Table 1) $\delta_{\mathrm{HA}}$ was almost constant, which fact indicates that there is probably no complexity between $M$ and HA. The values for $\delta_{\mathrm{HA}}$ used in the following calculations were those reported for $C_{\mathrm{M}}$ $=0, C_{\mathrm{HA}}=50 \mathrm{mM}$.

Table 1. Molar rotations of mandelic acid (degr. $\mathbf{M}^{-1} \mathrm{dm}^{-1}$ ).

\begin{tabular}{|c|c|c|c|c|c|}
\hline \multicolumn{6}{|c|}{$C_{\mathrm{HA}}=50 \mathrm{mM}$} \\
\hline$C_{\mathrm{M}} \mathrm{mM}$ & $-\delta_{\text {SBO }}$ & $-\delta_{578}$ & $-\delta_{540}$ & $-\delta_{\mathbf{4 s 6}}$ & $-\delta_{386}$ \\
\hline 0 & 24.5 & 25.6 & 29.4 & 53.2 & 90.5 \\
\hline 50 & 24.5 & 25.6 & 29.4 & 53.2 & 90.5 \\
\hline 100 & 24.6 & 25.7 & 29.5 & 53.3 & 90.7 \\
\hline 200 & 24.6 & 25.7 & 29.6 & 53.4 & 90.8 \\
\hline \multicolumn{6}{|c|}{$C_{\mathrm{M}}=25 \mathrm{mM}$} \\
\hline$C_{\mathrm{HA}} \mathrm{mM}$ & $-\delta_{580}$ & $-\delta_{578}$ & $-\delta_{548}$ & $-\delta_{\mathbf{A 3 6}}$ & $-\delta_{365}$ \\
\hline 25 & 24.6 & 25.7 & 29.5 & 53.2 & 90.5 \\
\hline 50 & 24.6 & 25.7 & 29.5 & 53.3 & 90.6 \\
\hline 100 & 24.6 & 25.8 & 29.6 & 53.4 & 90.8 \\
\hline
\end{tabular}

Acta Chem. Scand. 19 (1965) No. 1 
Table 2. $-\delta_{\mathrm{A}}$ as a function of $C_{\mathrm{A}}(\mathrm{mM})$. The ionic strength $=2 \mathrm{M}\left(\mathrm{NaClO}_{4}\right)$.

\begin{tabular}{|c|c|c|c|c|c|}
\hline \multirow{2}{*}{$\underset{\mathrm{m} \mathbf{M}}{C_{\mathrm{A}}}$} & \multicolumn{5}{|c|}{$-\delta_{\mathrm{A}} \operatorname{degr} . \mathrm{M}^{-1} \mathrm{dm}^{-1}$} \\
\hline & $589 \mathrm{~m} \mu$ & $578 \mathrm{~m} \mu$ & $546 \mathrm{~m} \mu$ & $436 \mathrm{~m} \mu$ & $365 \mathrm{~m} \mu$ \\
\hline $\begin{array}{r}\mathbf{2 5} \\
50 \\
100 \\
150 \\
200 \\
300\end{array}$ & $\begin{array}{l}18.55 \\
18.54 \\
18.60 \\
18.64 \\
18.68 \\
18.76\end{array}$ & $\begin{array}{l}19.39 \\
19.36 \\
19.44 \\
19.49 \\
19.54 \\
19.61\end{array}$ & $\begin{array}{l}22.23 \\
22.22 \\
22.31 \\
22.35 \\
22.41 \\
22.51\end{array}$ & $\begin{array}{l}39.82 \\
39.95 \\
40.06 \\
40.17 \\
40.27 \\
40.43\end{array}$ & $\begin{array}{l}67.09 \\
67.31 \\
67.50 \\
67.69 \\
67.86 \\
68.13\end{array}$ \\
\hline
\end{tabular}

The quantity $\delta_{\mathrm{A}}$, the molar rotation of $\mathrm{A}$, was determined for increasing $C_{\mathrm{A}}$. It was found (Table 2) to increase almost linearly with $C_{\mathrm{A}}$. The values of $\delta_{\mathrm{A}}$ used below are obtained by graphical interpolation of the values of Table 2.

In the main series of measurement $C_{\mathrm{M}}$ was kept constant and equal to 10 , 25 , and $50 \mathrm{~m} \mathrm{M}$, respectively. Unfortunately, when a certain $C_{\mathrm{A}}$ was reached, a precipitation, probably zinc mandelate, was formed. For this reason $C_{\mathrm{A}}$ was $\leq 200 \mathrm{mM}$ for $C_{\mathrm{M}}=10 \mathrm{mM}$ and $\leq 100 \mathrm{mM}$ for $C_{\mathrm{M}}=50 \mathrm{mM}$. Even many of these systems were not thermodynamically stable but formed supersaturated solutions. By rapidly mixing thermostated solutions it was possible to measure the rotation before precipitation occurred. The rate of precipitation increased with increasing $C_{\mathrm{A}}$ and $C_{\mathrm{M}}$; a solution of the composition $C_{\mathrm{M}}=$

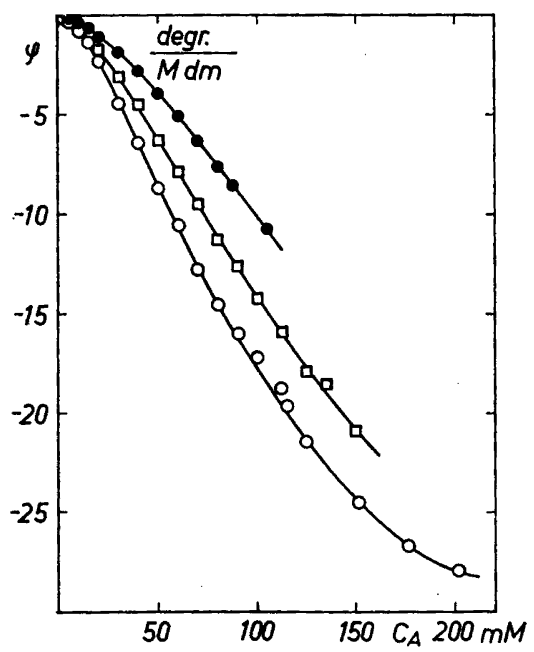

Fig. 1. The function $\varphi$ (eqn. (3)) as a function of $C_{\mathrm{A}}$ for different values of $C_{\mathrm{M}}$. $C_{\mathrm{M}}=10 \mathrm{mM}$, (O); $C_{\mathrm{M}}=25 \mathrm{mM}$, ( $\left.\square\right)$; $C_{\mathrm{M}}=50 \mathrm{mM}$, (O). $\lambda=546 \mathrm{~m} \mu$.

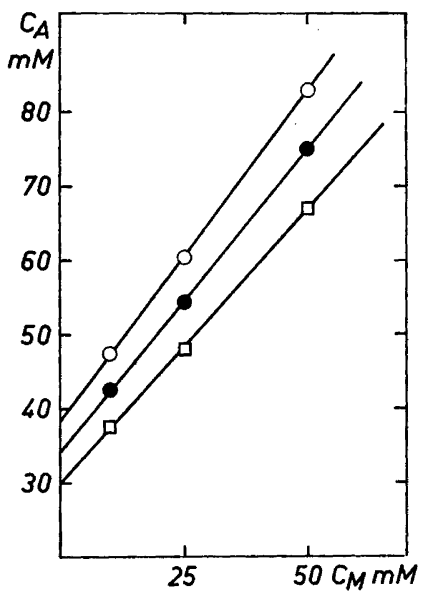

Fig. 2. Determination of corresponding values of $\bar{n}$ and $[A] . \lambda=546 \mathrm{~m} \mu . \varphi=-8$, (O); $\varphi=-7,(O) ; \varphi=-6,(\square) . \varphi$ in degr. $\mathbf{M}^{-1} \mathrm{dm}^{-1}$.

Acta Chem. Scand. 19 (1965) No. 1 
Table 3. Survey of measurements. Concentrations in $\mathrm{mM}, \vartheta^{\prime}$ in degrees and $\varphi$ in degrees $\mathrm{M}^{-1} \mathrm{dm}^{-1}$.

$\lambda=589 \mathrm{~m} \mu, C_{\mathrm{M}}=10 \mathrm{mM}$

$C_{\mathrm{A}^{\prime}},-\vartheta^{\prime},-\varphi: 2,0.039,0.15 ; 5,0.097_{5}, 0.37 ; 10,0.196,0.83 ; 15,0.295,1.35 ; 20,0.394_{5}$, $1.88 ; 30,0.603,3.95 ; 40,0.806,5.42 ; 50,1.012,7.18 ; 60,1.216 ; 8.76 ; 70,1.425,10.73$; $80,1.629_{5}, 12.35 ; 90,1.828_{5}, 13.25 ; 100,2.027_{5}, 14.30 ; 112.5,2.279,15.79 ; 115,2.332$, 16.38 ; $125,2.537,17.89 ; 151.3,3.060,20.36$; 176.5, 3.559, 22.31; 201.7, 4.051, 23.46.

$\lambda=578 \mathrm{~m} \mu, C_{\mathrm{M}}=10 \mathrm{mM}$

$C_{\mathrm{A}^{\prime}},-\vartheta^{\prime},-\varphi: 2,0.041,0.18 ; 5,0.101_{5}, 0.36 ; 10,0.203_{5}, 0.75 ; 15,0.306_{5}, 1.25 ; 20,0.413$, $2.07 ; 30,0.628_{5}, 3.98 ; 40,0.841_{5}, 5.61 ; 50,1.058_{5}, 7.62 ; 60,1.271_{5}, 9.22 ; 70,1.487,11.04$; $80,1.701,12.69 ; 90,1.910_{5}, 13.88 ; 100,2.119,14.94 ; 112.5,2.380_{5}, 16.36 ; 115,2.437$, $16.97 ; 125,2.651,18.53 ; 151.3,3.198,21.13 ; 176.5,3.720,23.02 ; 201.7,4.234,24.19$.

$\lambda=546 \mathrm{~m} \mu, C_{\mathrm{M}}=10 \mathrm{mM}$

$C_{\mathrm{A}^{\prime}},-\vartheta^{\prime},-\varphi: 2,0.046_{5}, 0.16 ; 5,0.116,0.36 ; 10,0.233,0.83 ; 15,0.351,1.37 ; 20,0.473_{5}$, $2.35 ; 30,0.720,4.47 ; 40,0.965,6.41 ; 50,1.213_{5}, 8.69 ; 60,1.458,10.54 ; 70,1.706_{5}, 12.78$; $80,1.950_{5}, 14.55 ; 90,2.192_{5}, 16.00 ; 100,2.431_{5}, 17.22 ; 112.5,2.730_{5}, 18.76 ; 115,2.796$, $19.66 ; 125,3.041,21.43 ; 151.3,3.670,24.51 ; 176.5,4.268,26.69 ; 207.1,4.858,27.95$.

$\lambda=436 \mathrm{~m} \mu, C_{\mathrm{M}}=10 \mathrm{mM}$

$C_{\mathrm{A}^{\prime}},-\vartheta^{\prime},-\varphi: 2,0.081,0.04 ; 5,0.205_{5}, 0.37 ; 10,0.415_{5}, 1.16 ; 15,0.628_{5}, 2.25 ; 20,0.845$, $3.67 ; 30,1.287_{5}, 7.47 ; 40,1.728,11.01 ; 50,2.176,15.20 ; 60,2.612,18.20 ; 70,3.059_{5}, 22.31$; $80,3.497,25.38 ; 90,3.928_{5}, 27.72 ; 100,4.357,29.80 ; 112.5,4.894_{5}, 32.47 ; 115,5.011_{5}$, $33.90 ; 125,5.450_{5}, 36.92$; $151.3,6.579,42.28 ; 176.5,7.652,46.09 ; 201.7,8.711,48.29$.

$\lambda=365 m \mu, C_{\mathrm{M}}=10 \mathrm{mM}$

$C_{\mathrm{A}^{\prime}},-\vartheta^{\prime}, 2,2,0.135_{5}, 0 ; 5,0.344_{5}, 0.45 ; 10,0.697,1.66 ; 15,1.054,3.29 ; 20,1.416$, $5.41 ; 30,2.10 \mathrm{~L}, 1.82 ; 40,2.899,17.18 ; 50,3.649_{5}, 23.90 ; 60,4.383_{5}, 28.84 ; 70,5.137$, $35.66 ; 80,5.873,40.72 ; 90,6.599,44.55 ; 100,7.319,47.87 ; 112.5,8.225,52.40 ; 115,8.421$, $54.78 ; 125,9.161_{5}, 59.99 ; 151.3,11.055,68.00 ; 176.5,12.860,73.98 ; 201.7,14.642,77.49$.

$\lambda=589 \mathrm{~m} \mu, C_{\mathrm{M}}=25 \mathrm{mM}$

$C_{\mathrm{A}^{\prime}},-\vartheta^{\prime},-\varphi: 2,0.040_{5}, 0.12 ; 5,0.101,0.29 ; 10,0.200,0.49 ; 15,0.305,0.94 ; 20,0.411$, $1.41 ; 30,0.628,2.58 ; 40,0.847,3.81 ; 50,1.071_{5}, 5.25 ; 60,1.293_{5}, 6.58 ; 70,1.516,7.93$; $80,1.742,9.44 ; 90,1.959,10.52 ; 100,2.183,11.94 ; 112.5,2.454,13.32 ; 125,2.731_{5}, 14.94$; $135,2.935,15.48 ; 150,3.270,17.49$.

$\lambda=578 \mathrm{~m} \mu, C_{\mathrm{M}}=25 \mathrm{mM}$

$C_{\mathrm{A}^{\prime}},-\vartheta^{\prime},-\varphi: 2,0.043,0.15 ; 5,0.105_{5}, 0.30 ; 10,0.210,0.56 ; 15,0.318_{5}, 0.98 ; 20,0.430$, $1.51 ; 30,0.657,2.73 ; 40,0.884,3.94 ; 50,1.118_{5}, 5.45 ; 60,1.350_{5}, 6.85 ; 70,1.583_{5}, 8.28$; $80,1.819,9.80 ; 90,2.047,11.01 ; 100,2.279_{5}, 12.40 ; 112.5,2.564_{5}, 13.92 ; 125,2.855,15.57$; $135,3.068 ; 16.14 ; 150,3.419,18.24$.

$\lambda=546 \mathrm{~m} \mu, C_{\mathrm{M}}=25 \mathrm{mM}$

$C_{\mathrm{A}^{\prime}},-\vartheta^{\prime},-\varphi: 2,0.048,0.12 ; 5,0.120_{5}, 0.32 ; 10,0.240_{5}, 0.63 ; 15,0.365_{5}, 1.13 ; 20,0.493$, $1.72 ;, 30,0.752_{5}, 3.09 ; 40,1.012_{5}, 4.46 ; 50,1.282_{5}, 6.24 ; 60,1.549,7.86 ; 70,1.816_{5}, 9.51$; $80,2.086,11.24 ; 90,2.347,12.58 ; 100,2.613_{5}, 14.17 ; 112.5,2.941_{5}, 15.94 ; 125,3.272_{5}$, $17.83 ; 135,3.518,18.50 ; 150,3.918,20.86$.

$\lambda=436 \mathrm{~m} \mu, C_{\mathrm{M}}=25 \mathrm{mM}$

$C_{\mathrm{A}^{\prime}},-\vartheta^{\prime},-\varphi: 2,0.087,0.26 ; 5,0.215_{5}, 0.55 ; 10,0.429_{5}, 1.02 ; 15,0.652,1.84 ; 20,0.880$, $2.87 ; 30,1.344,5.25 ; 40,1.810_{5}, 7.70 ; 50,2.294_{5}, 10.82 ; 60,2.770,13.60 ; 70,3.247_{5}, 16.44$; $80,3.731,19.51 ; 90,4.196,21.79 ; 100,4.672_{5}, 24.54 ; 112.5,5.258,27.53 ; 125,5.854_{5}$, $30.93 ; 135,6.294,32.14 ; 150,7.008,36.12$.

$\lambda=365 \mathrm{~m} \mu, C_{\mathrm{M}}=25 \mathrm{mM}$

$C_{\mathrm{A}^{\prime}},-\vartheta^{\prime},-\varphi: 2,0.143,0.29 ; 5,0.357_{5}, 0.70 ; 10,0.715_{5}, 1.40 ; 15,1.087_{5}, 2.66 ; 20,1.467_{5}$, $4.22 ; 30,2.247,8.13 ; 40,3.026_{5}, 11.97 ; 50,3.836_{5}, 17.04 ; 60,4.630_{5}, 21.42 ; 70,5.431_{5}$, 
$26.04 ; 80,6.242,31.05 ; 90,7.017,34.54 ; 100,7.820,39.19 ; 112.5,8.802,44.04 ; 125,9.802_{5}$, $49.64 ; 135,10.535,51.32 ; 150,11.738,57.96$.

$\lambda=589 \mathrm{~m} \mu, C_{\mathrm{M}}=50 \mathrm{mM}$

$C_{\mathrm{A}^{\prime}},-\vartheta^{\prime},-\varphi: 2,0.041,0.07 ; 5,0.100_{5}, 0.13 ; 10,0.205,0.35 ; 15,0.310_{5}, 0.58 ; 20,0.422$, $0.93 ; 30,0.641_{5}, 1.56 ; 40,0.871,2.38 ; 50,1.105,3.30 ; 60,1.341_{5}, 4.25 ; 70,1.581,5.27$; $80,1.825,6.38 ; 87.5,2.005,7.14 ; 105,2.430,9.01$.

$\lambda=578 \mathrm{~m} \mu, C_{\mathrm{M}}=50 \mathrm{mM}$

$C_{\mathrm{A}}{ }^{\prime}-\vartheta^{\prime},-\varphi: 2,0.043,0.08 ; 5,0.104_{5}, 0.13 ; 10,0.213,0.34 ; 15,0.322_{5}, 0.57 ; 20,0.440_{5}$, $0.96 ; 30,0.669_{5}, 1.62 ; 40,0.908_{5}, 2.46 ; 50,1.155,3.45 ; 60,1.401_{5}, 4.44 ; 70,1.652,5.51$; $80,1.908,6.68 ; 87.5,2.095,7.45 ; 105,2.539,9.40$.

$\lambda=546 \mathrm{~m} \mu, C_{\mathrm{M}}=50 \mathrm{mM}$

$C_{A^{\prime}},-\vartheta^{\prime},-\varphi: 2,0.049_{5}, 0.09 ; 5,0.120,0.15 ; 10,0.244,0.39 ; 15,0.370,0.65 ; 20,0.505$; $1.10 ; 30,0.767,1.83 ; 40,1.040_{5}, 2.79 ; 50,1.323_{5}, 3.94 ; 60,1.606,5.07 ; 70,1.894,6.31$; $80,2.187,7.64 ; 87.5,2.401,8.52 ; 105,2.911,10.77$.

$\lambda=436 \mathrm{~m} \mu, C_{\mathrm{M}}=50 \mathrm{mM}$

$C_{\mathrm{A}^{\prime}},-\vartheta^{\prime},-\varphi: 2,0.088,0.15 ; 5,0.215,0.26 ; 10,0.435_{5}, 0.63 ; 15,0.659_{5}, 1.07 ; 20,0.900_{\mathrm{s}}$, $1.84 ; 30,1,367_{5}, 3.09 ; 40,1.856_{5}, 4.77 ; 50,2.361,6.74 ; 60,2.865_{5}, 8.71 ; 70,3.377,10.81 ;$ $80,3.898,13.10 ; 87.5,4.282,14.66 ; 105,5.189,18.52$.

$\lambda=365 \mathrm{~m} \mu, C_{\mathrm{M}}=50 \mathrm{mM}$

$C_{\mathrm{A}^{\prime}},-\vartheta^{\prime},-\varphi: 2,0.145,0.18 ; 5,0.356,0.32 ; 10,0.723_{5}, 0.86 ; 15,1.097,1.52 ; 20,1.498$, $2.72 ; 30,2.277_{5}, 4.67 ; 40,3.092,7.30 ; 50,3.931_{5}, 10.42 ; 60,4.772,13.54 ; 70,5.625,16.89$; $80,6.494,20.56 ; 87.5,7.134,23.06 ; 105,8.651,29.33$.

Table 4. Experimental values of $\bar{n}$.

\begin{tabular}{|c|c|c|c|c|c|c|}
\hline \multirow{2}{*}{$\begin{array}{l}{[\mathrm{A}]} \\
\mathbf{m M}\end{array}$} & \multicolumn{5}{|c|}{$\bar{n}$} & \multirow{2}{*}{$\bar{n}_{\text {mean }}$} \\
\hline & $589 \mathrm{~m} \mu$ & $578 \mathrm{~m} \mu$ & $546 \mathrm{~m} \mu$ & $436 \mathrm{~m} \mu$ & $365 \mathrm{~m} \mu$ & \\
\hline 5 & 0.14 & 0.15 & 0.15 & 0.14 & 0.10 & 0.14 \\
\hline 10 & 0.29 & 0.27 & 0.25 & 0.25 & 0.23 & 0.26 \\
\hline 15 & 0.40 & 0.39 & 0.40 & 0.37 & 0.35 & $\mathbf{0 . 3 8}$ \\
\hline 20 & 0.55 & 0.53 & 0.52 & 0.50 & 0.48 & 0.52 \\
\hline 25 & 0.66 & 0.64 & 0.64 & 0.61 & 0.63 & 0.64 \\
\hline 30 & 0.76 & 0.74 & 0.75 & 0.71 & 0.75 & 0.74 \\
\hline 35 & 0.83 & 0.82 & 0.84 & 0.82 & 0.85 & 0.83 \\
\hline 40 & 0.92 & 0.91 & 0.93 & 0.94 & 0.96 & 0.93 \\
\hline 45 & 1.01 & 1.00 & 1.02 & 1.06 & 1.07 & 1.03 \\
\hline 50 & 1.10 & 1.11 & 1.12 & 1.18 & 1.18 & 1.14 \\
\hline 55 & 1.26 & 1.24 & 1.22 & 1.30 & 1.30 & 1.26 \\
\hline 60 & 1.34 & 1.33 & 1.34 & 1.40 & 1.46 & 1.37 \\
\hline 65 & 1.40 & 1.37 & 1.43 & 1.48 & 1.56 & 1.45 \\
\hline 70 & 1.46 & 1.43 & 1.53 & 1.55 & 1.62 & $\mathbf{1 . 5 2}$ \\
\hline 75 & 1.55 & 1.50 & 1.60 & 1.62 & 1.68 & 1.59 \\
\hline 80 & 1.65 & 1.55 & 1.67 & 1.68 & 1.72 & 1.65 \\
\hline 85 & 1.73 & 1.63 & 1.75 & 1.75 & 1.78 & 1.73 \\
\hline 90 & 1.78 & 1.67 & 1.82 & 1.80 & 1.83 & 1.78 \\
\hline 95 & 1.83 & 1.72 & 1.88 & 1.85 & 1.88 & 1.83 \\
\hline 100 & 1.88 & 1.78 & 1.93 & 1.88 & 1.94 & 1.88 \\
\hline
\end{tabular}

Acta Chem. Scand. 19 (1965) No. 1 
$25 \mathrm{mM}, C_{\mathrm{A}}=20 \mathrm{mM}$ did form a precipitate after some weeks. All solutions were carefully checked for any presence of turbidity before and after every measurement.

In Table 3 we give the experimental data relevant for a calculation of $\bar{n}$.

For each wavelength $\varphi$ was then plotted against $C_{\mathrm{A}}\left(=C_{\mathrm{A}}{ }^{\prime}+\left[\mathrm{H}^{+}\right]\right)$. Fig. 1 gives a representative case, $\lambda=546 \mathrm{~m} \mu$. It can be seen from this curve that it is not possible to obtain $\bar{n}$-values for [A] $>$ about $100 \mathrm{mM}$. This, of course, is due to the fact that it was not possible to extend the measurements very far for $C_{\mathrm{M}}=25$ and $50 \mathrm{mM}$.

As described above, the $\varphi$-curves were cut at fixed values of $\varphi$ and $C_{\mathrm{A}}$ was plotted against $C_{\mathrm{M}}$. Fig. 2 gives some examples of this procedure. Hence, for each wavelength a set of ([A], $\bar{n})$ pairs was obtained.

By graphical interpolation a set of $\bar{n}$-values for a series of values of [A] was then obtained for the different wavelengths. These are given in Table 4 together with the mean value of $\bar{n}$.

\section{CALCULATION OF THE' STABILITY CONSTANTS}

In order to obtain the $\mathrm{X}$-function from the $(\bar{n},[\mathrm{~A}])$ pairs we formed the quantity $\bar{n} /[\mathrm{A}]$. It holds ${ }^{2,6}$ that

$\ln \mathbf{X}\left([\mathbf{A}]_{\mathrm{i}}\right)=\int_{0}^{[\mathrm{A}]}(\bar{n} /[\mathbf{A}]) \mathrm{d}[\mathbf{A}]$

The integration was performed graphically from a $\bar{n} /[\mathrm{A}]$ versus [A] plot.

Table 5. Calculation of the stability constants. The following values are obtained: $\beta_{1}=$ $30 \mathrm{M}^{-1}, \beta_{2}=260 \mathrm{M}^{-2}, \beta_{3}=3870 \mathrm{M}^{-3}$.

\begin{tabular}{|c|c|c|c|c|c|c|}
\hline $\begin{array}{l}\text { [A] } \\
\mathbf{m M}\end{array}$ & $\begin{array}{c}\bar{n} /[\mathbf{A}] \\
\left.\mathbf{M}^{-1}\right]\end{array}$ & $\mathbf{X}$ & $X_{1}$ & $\mathbf{X}_{2}$ & $\mathbf{X}_{3}$ & $\bar{n}_{\text {calc }}$ \\
\hline 5 & 28.0 & $1.15_{7}$ & 31.4 & 280 & & 0.14 \\
\hline 10 & 27.5 & $1.33_{1}$ & 33.1 & 310 & & 0.27 \\
\hline 15 & 26.7 & 1.52 。 & 34.9 & 327 & & 0.40 \\
\hline 20 & 26.0 & $1.73_{8}$ & 36.9 & 345 & & 0.52 \\
\hline 25 & 25.6 & 1.97 , & 39.1 & 364 & & 0.64 \\
\hline 30 & 25.0 & 2.24 & 41.4 & 380 & & 0.75 \\
\hline 35 & 24.6 & 2.54 & 43.9 & 397 & & 0.86 \\
\hline 40 & 24.3 & 2.87 & 46.6 & 415 & 3880 & 0.97 \\
\hline 45 & 23.8 & 3.23 & 49.5 & 433 & $\mathbf{3 8 5 0}$ & 1.07 \\
\hline 50 & 23.4 & 3.63 & 52.7 & 454 & 3880 & 1.17 \\
\hline 55 & 23.1 & 4.08 & 56.0 & 473 & 3870 & 1.26 \\
\hline 60 & 22.7 & 4.57 & 59.5 & 492 & 3860 & 1.35 \\
\hline 65 & 22.2 & 5.11 & 63.3 & 512 & 3880 & 1.44 \\
\hline 70 & 21.7 & 5.70 & 67.2 & 531 & 3880 & 1.51 \\
\hline 75 & 21.2 & 6.35 & 71.3 & 551 & 3880 & 1.59 \\
\hline 80 & 20.8 & 7.05 & 75.6 & 570 & 3880 & 1.66 \\
\hline 85 & 20.2 & 7.81 & 80.1 & 589 & 3880 & 1.72 \\
\hline 90 & 19.8 & 8.62 & 84.7 & 608 & 3860 & 1.78 \\
\hline 95 & 19.3 & 9.51 & 89.6 & 627 & $\mathbf{3 8 7 0}$ & 1.84 \\
\hline 100 & 18.8 & 10.46 & 94.6 & 646 & 3860 & 1.89 \\
\hline
\end{tabular}

Acta Chem. Scand. 19 (1965) No. 1 
The results are given in Table 5 where also $\overline{\mathbf{X}}_{n}$ is included. $\overline{\mathrm{X}}_{n}$ is defined by the formula 2,6

$$
\mathrm{X}_{n+1}=\left(\mathrm{X}_{n}-\beta_{n}\right) /[\mathrm{A}]
$$

where $\beta_{0}=1$

It is obvious from relations (5) and (9) that $\beta_{n}=\lim _{[A] \rightarrow 0} \mathrm{X}_{n}$.

The constancy of $\mathrm{X}_{3}$ indicates that three and not more than three complexes exist, at least not within the [A]-range investigated.

From the values of the stability constants thus obtained $\bar{n}$ has been calculated from eqn. (6). The calculated values of $\bar{n}$ are included in Table 5. It can be seen that there is a close agreement with the experimental values of Table 4. This makes it probable that the calculation of the complexity constants is correct.

\section{THE MOLAR ROTATIONS OF THE COMPLEXES}

Eqn. (4) can be rewritten in the form

$$
\varphi=\sum_{n=1}^{N} \alpha_{n}\left(\delta_{n}-n \delta_{\mathrm{A}}\right)
$$

where $\alpha_{n}$ is the fraction $\left[\mathrm{MA}_{n}\right] / C_{\mathrm{M}}$. For the sake of brevity we define:

$$
D_{n}=\delta_{n}-n \delta_{\Lambda}
$$

Now, from the stability constants we can calculate $\alpha_{n}$ and then obtain $D_{n}$.

\begin{tabular}{|c|c|c|c|c|c|c|c|c|c|c|c|c|c|c|c|}
\hline \multirow{2}{*}{$\begin{array}{l}{[\mathbf{A}]} \\
\mathbf{m M}\end{array}$} & \multicolumn{3}{|c|}{$589 \mathrm{~m} \mu$} & \multicolumn{3}{|c|}{$578 \mathrm{~m} \mu$} & \multicolumn{3}{|c|}{$546 \mathrm{~m} \mu$} & \multicolumn{3}{|c|}{$436 \mathrm{~m} \mu$} & \multicolumn{3}{|c|}{$365 \mathrm{~m} \mu$} \\
\hline & $-\varphi$ & $-\varphi^{\prime}$ & $-\varphi^{\prime \prime}$ & $-\varphi$ & $-\varphi^{\prime}$ & $-\varphi^{\prime \prime}$ & $-\varphi$ & $-\varphi^{\prime}$ & $-\varphi^{\prime \prime}$ & $-\varphi$ & $-\varphi^{\prime}$ & $-\varphi^{\prime \prime}$ & $-\varphi$ & $-\varphi^{\prime}$ & $-\varphi^{\prime \prime}$ \\
\hline 5 & 0.4 & 3.1 & & 0.5 & 3.7 & & 0.4 & 3.3 & & 0.7 & 5.0 & & 0.7 & 5.0 & \\
\hline 10 & 1.1 & 4.9 & & 1.1 & 5.0 & & 1.2 & 5.3 & & 1.7 & 7.5 & & 2.2 & 9.5 & \\
\hline 15 & 1.9 & 6.4 & & 2.0 & 6.6 & & 2.3 & 7.7 & & 3.4 & 11.5 & & 5.0 & 16.7 & \\
\hline 20 & 2.9 & 8.5 & & 3.0 & 8.7 & & 3.5 & 10.1 & & 5.7 & 16.3 & & 8.6 & 24.9 & \\
\hline 25 & 4.0 & 10.5 & & 4.1 & 10.8 & & 4.8 & 12.5 & & 7.9 & 20.8 & & 12.2 & 32.1 & \\
\hline 30 & 5.0 & 12.4 & & 5.2 & 12.8 & & 6.0 & 14.9 & & 10.2 & 25.3 & & 15.9 & 39.6 & \\
\hline 35 & 6.0 & 14.5 & & 6.2 & 15.0 & & 7.3 & 17.5 & & 12.4 & 30.0 & & 19.6 & 47.3 & \\
\hline 40 & 7.1 & 16.8 & 376 & 7.3 & 17.4 & 364 & 8.5 & 20.2 & 464 & 14.7 & 35.0 & 804 & 23.2 & 55.4 & 1359 \\
\hline 45 & 8.1 & 19.4 & 391 & 8.3 & 19.9 & 380 & 9.7 & 23.1 & 478 & 16.9 & 40.4 & 836 & 26.9 & 64.4 & 1408 \\
\hline 50 & 9.1 & 22.0 & 405 & 9.4 & 22.7 & 399 & 10.8 & 26.3 & 493 & 19.2 & 46.5 & 874 & 30.6 & 74.1 & 1462 \\
\hline 55 & 10.1 & 25.0 & 422 & 10.4 & 25.7 & 417 & 12.0 & 29.8 & 512 & 21.3 & 52.7 & 908 & 34.2 & 84.7 & 1521 \\
\hline 60 & 11.0 & 27.9 & 438 & 11.3 & 28.8 & 433 & 13.2 & 33.5 & 531 & 23.2 & 58.9 & 935 & 37.6 & 95.4 & 1574 \\
\hline 65 & 11.9 & 31.2 & 452 & 12.2 & 32.0 & 449 & 14.3 & 37.3 & 549 & 25.0 & 65.5 & 964 & 40.6 & 106.3 & 1620 \\
\hline 70 & 12.8 & $\mathbf{3 4 . 8}$ & 471 & 13.1 & 35.6 & 469 & 15.3 & 41.6 & 571 & 26.7 . & 72.4 & 995 & 43.3 & 117.7 & 1667 \\
\hline 75 & 13.6 & 38.3 & 487 & 13.9 & 39.2 & 485 & 16.3 & 45.9 & 591 & 28.3 & 79.6 & 1024 & 45.9 & 129.3 & 1711 \\
\hline 80 & 14.4 & 42.2 & 505 & 14.7 & 43.1 & 504 & 17.3 & 50.6 & 612 & 29.8 & 87.4 & 1057 & 48.4 & 141.9 & 1762 \\
\hline 85 & 15.2 & 46.3 & 524 & 15.5 & 47.3 & 524 & 18.2 & 55.6 & 635 & 31.3 & 95.7 & 1093 & 50.8 & 155.4 & 1816 \\
\hline 90 & 15.9 & 50.6 & 543 & 16.2 & 51.7 & 544 & 19.1 & 60.9 & 658 & 32.8 & 104.6 & 1131 & 53.1 & 169.7 & 1874 \\
\hline 95 & 16.5 & 55.0 & 560 & 16.9 & 56.2 & 562 & 19.8 & 66.1 & 679 & 34.1 & 113.7 & 1167 & 55.3 & 184.3 & 1930 \\
\hline 100 & 17.1 & 59.6 & 578 & 17.5 & 61.1 & 583 & 20.6 & 71.8 & 702 & 35.4 & 123.2 & 1204 & 57.5 & 200.2 & 1992 \\
\hline
\end{tabular}
This is done in the following way:

Table 6. Determination of the entities $\varphi^{\prime}\left(\operatorname{degr} . \mathrm{M}^{-1} \mathrm{dm}^{-1}\right)$ and $\varphi^{\prime \prime}\left(\operatorname{degr} . \mathrm{M}^{-2} \mathrm{dm}^{-1}\right)$.

Acta Chem. Scand. 19 (1965) No. 1 
Table 7. Molar rotations (degr. $\mathrm{M}^{-1} \mathrm{dm}^{-1}$ ) of the complexes.

\begin{tabular}{|c|r|r|r|r|r|r|r|r|c|}
\hline $\begin{array}{c}\text { Wave- } \\
\text { length } \mathrm{m} \mu\end{array}$ & $-D_{1}$ & $-D_{2}$ & $-D_{3}$ & $-\delta_{1}$ & $-\delta_{2}$ & $-\delta_{3}$ & $-\delta_{2} / 2$ & $-\delta_{3} / 3$ & $-\delta_{\mathrm{A}}$ \\
\hline 589 & 1.8 & 25.6 & 27.6 & 20.3 & 62.6 & 83.1 & 31.3 & 27.7 & 18.5 \\
578 & 2.8 & 24.8 & 28.1 & 22.1 & 63.4 & 86.0 & 31.7 & 28.7 & 19.3 \\
546 & 1.6 & 30.7 & 33.8 & 23.8 & 75.1 & 100.4 & 37.6 & 33.5 & 22.2 \\
436 & 2.8 & 62.9 & 50.2 & 42.6 & 142.5 & 169.6 & 71.3 & 56.5 & 39.8 \\
365 & 1.0 & 105.6 & 82.6 & 68.1 & 239.8 & 283.9 & 119.9 & 94.6 & 67.1 \\
\hline
\end{tabular}

$\alpha_{1}, \alpha_{2}$ and $\alpha_{3}$ was calculated for a series of even values of [A] and the function $\varphi^{\prime}=\varphi / \alpha_{1}$ was plotted against [A].

From eqns. (10) and (11) it follows that

$$
\lim _{[\mathrm{A}] \rightarrow 0} \varphi^{\prime}=D_{1}
$$

Then the function $\varphi^{\prime \prime}=\left(\varphi^{\prime}-D_{1}\right) /[\mathrm{A}]$ was formed.

One immediately obtains

$$
\varphi^{\prime \prime}=\left(\beta_{2} / \beta_{1}\right) D_{2}+\left(\beta_{3} / \beta_{1}\right) D_{3}[\mathrm{~A}]
$$

$\varphi^{\prime \prime}$ was plotted against [A] and a straight line was obtained. From the intercept of this line $D_{2}$ has been determined and from the slope one gets $D_{3}$. The results are given in Tables 6 and 7 . An example of the $\varphi^{\prime \prime}$ versus [A] plot is given in Fig. 3.

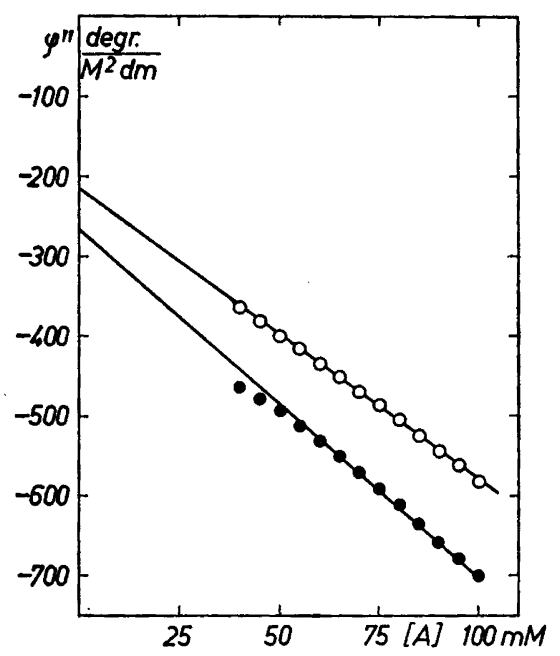

Fig. 3. An example of the determination of $D_{2}$ and $D_{3}$. The upper curve represents the values for wavelength $578 \mathrm{~m} \mu$, the lower one $546 \mathrm{~m} \mu$. 
Table 8. Determination of the wavelength $\lambda_{0 n}$.

\begin{tabular}{|l|c|c|c|c|c|}
\hline$\lambda \mathrm{m} \mu$ & $\lambda^{2} \times 10^{-4}$ & $\frac{-10^{3}}{\delta_{\mathrm{A}}}$ & $\frac{-10^{3}}{\delta_{1}}$ & $\frac{-10^{3}}{\delta_{2}}$ & $\frac{-10^{3}}{\delta_{3}}$ \\
\hline 589 & 34.69 & 54.1 & 49.3 & 16.0 & 12.0 \\
578 & 33.40 & 51.8 & 45.2 & 15.8 & 11.6 \\
546 & 29.81 & 45.0 & 42.0 & 13.3 & 10.0 \\
436 & 19.01 & 25.1 & 23.5 & 7.0 & 5.9 \\
365 & $5.3 \pm 0.1$ & 14.9 & 14.7 & 4.2 & 3.5 \\
$230 \pm 2$ & 0 & 0 & & \\
$210 \pm 8$ & $4.4 \pm 0.3$ & & & 0 & 0 \\
$255 \pm 8$ & $6.5 \pm 0.3$ & & & & 0 \\
$215 \pm 8$ & $4.6 \pm 0.3$ & & & & \\
\hline
\end{tabular}

The values of $\delta_{n}$ thus arrived at can be used for a determination of the wavelength of the electron transition that is causing the optical activity of the mandelate ion in the various complexes. If this wavelength is called $\lambda_{0 n}$ the Drude ${ }^{10}$ equation yields

$$
\delta_{n}=K\left(\lambda^{2}-\lambda_{0 n}{ }^{2}\right)^{-1}
$$

This equation can be transformed to

$$
1 / \delta_{n}=(1 / K)\left(\lambda^{2}-\lambda_{0 n}{ }^{2}\right)
$$

A plot of $1 / \delta_{n}$ versus $\lambda^{2}$ will then give $\lambda_{0 n^{2}}=\left(\lambda^{2}\right)_{1 / \delta_{n}=0}$.

These plots were found to be perfectly straight lines and $\lambda_{0 n}$ could be determined within reasonably close limits (Table 8). The application of the Drude equation is justified since all measurements were made at wavelengths far away from the absorption region.

In Table 7 there are also included the molar rotations per ligand of the complexes, i.e. $\delta_{n} / n$.

\section{DISCUSSION}

From the reported data one can calculate the consecutive stability constants $\left.k_{n}=\beta_{n} \mid \beta_{n-1}\right)$. One then obtains $k_{1}=30 \mathrm{M}^{-1}, k_{2}=8.7 \mathrm{M}^{-1}$, and $k_{3}$ $=15 \mathrm{M}^{-1}$. This gives $k_{1} / k_{2}=3.4$ and $k_{2} / k_{3}=0.6$. The first ratio is of the order of magnitude that may be expected ${ }^{11}$ if the ligands occupy only one coordination site each. The other ratio, on the other hand, is much smaller than what is usually found. This means that $\beta_{3}$ is unexpectedly large. One possibility to account for this is that when the third complex is formed, the coordination number of the central atom changes from six to four:

or

$$
\mathrm{Maq}_{4} \mathrm{~A}_{2}+\mathrm{A} \rightleftharpoons \mathrm{Maq} \mathrm{A}_{3}+3 \mathrm{aq}
$$

$$
\mathrm{Maq}_{4} \mathrm{~A}_{2}+\mathrm{A} \rightleftharpoons \mathrm{MA}_{3}+4 \mathrm{aq}
$$

In the last formula $\mathrm{MA}_{3}$ should mean that one ligand forms a chelate ring. In any of these ways the entropy increase resulting from the liberation of water should make $\beta_{3}$ larger than was expected from the values of $k_{1}$ and $k_{2}$. 
The change of the trends of the optical parameters $\lambda_{0 n}$ and $\delta_{n} / n$ from the second to the third complex also give evidence of some drastic change in the architecture of the complex. A full interpretation, however, must wait until the investigation of other metal-mandelate systems and also a check of the results with independent methods have been performed.

It would be especially interesting to measure the complexity of both optically active and inactive mandelate ions. Such work is planned at this laboratory.

\section{REFERENCES}

1. Woldbye, F. Acta Chem. Scand. 9 (1955) 299.

2. Fronæus, S. Komplexsystem hos koppar, Lund 1948.

3. Sonesson, A. Acta Chem. Scand. 13 (1959) 998.

4. Ahrland, S. Acta Chem. Scand. 7 (1953) 485.

5. Biedermann, G. \%. ICCC, p. 159.

6. Fronmus, S. Determinations of Formation Constants of Complexes, Chapt. 1, in Tech. nique of Inorg. Chem. Eds. Jonassen, H. B. and Weissberger, A. Vol. 1. 1963. Interscience Publishers.

7. Csiszar, B., Halmos, M. and Beck, M. T. Naturwiss. 48 (1961) 571.

8. Banks, W. H. and Davies, C. W. J. Chem. Soc. 193873.

9. Hope, D. A. L., Otter, R. J. and Prue, J. E. J. Chem. Soc. 19605226.

10. Drude, P. Lehrbuch der Optik, Leipzig 1900.

11. Bjerrum, J. Metal ammine formation in aqueous solution, Copenhagen 1941.

Received September 28, 1964. 